\title{
New Directions in Anti-Angiogenic Therapy for Glioblastoma
}

\author{
Nancy Wang ${ }^{1}$ - Rakesh K. Jain ${ }^{2}$ - Tracy T. Batchelor ${ }^{1,2,3}$
}

Published online: 12 January 2017

(C) The American Society for Experimental NeuroTherapeutics, Inc. 2017

\begin{abstract}
Anti-angiogenic therapy has become an important component in the treatment of many solid tumors given the importance of adequate blood supply for tumor growth and metastasis. Despite promising preclinical data and early clinical trials, anti-angiogenic agents have failed to show a survival benefit in randomized controlled trials of patients with glioblastoma. In particular, agents targeting vascular endothelial growth factor (VEGF) appear to prolong progression free survival, possibly improve quality of life, and decrease steroid usage, yet the trials to date have demonstrated no extension of overall survival. In order to improve duration of response and convey a survival benefit, additional research is still needed to explore alternative pro-angiogenic pathways, mechanisms of resistance, combination strategies, and biomarkers to predict therapeutic response.
\end{abstract}

Keywords Angiogenesis · Glioblastoma · Glioma ·

Bevacizumab · VEGF

\section{Introduction}

Angiogenesis is a hallmark of glioblastoma and remains an important therapeutic target in its treatment. Despite a

Tracy T. Batchelor

tbatchelor@mgh.harvard.edu

1 Stephen E. and Catherine Pappas Center for Neuro-Oncology, Massachusetts General Hospital, Boston, MA, USA

2 Department of Radiation Oncology, Massachusetts General Hospital, Boston, MA, USA

3 Department of Hematology/Oncology, Massachusetts General Hospital, Boston, MA, USA multimodality approach consisting of surgery in addition to radiation therapy with concurrent and adjuvant temozolomide, the prognosis for glioblastoma remains poor with a median survival of 14-16 months [1, 2]. Because glioblastomas are histologically characterized by microvascular proliferation and express high levels of pro-angiogenic factors such as vascular endothelial growth factor (VEGF), agents that target the VEGF-pathway have been investigated in hopes of expanding currently limited treatment options (Table 1).

Bevacizumab is a recombinant, humanized, monoclonal antibody targeting the VEGF ligand A that was first approved for the treatment of metastatic colorectal cancer by the United States Food and Drug Administration (FDA) in 2004. It was subsequently approved for the treatment of non-small cell lung cancer in 2006 , followed by renal cell carcinoma and recurrent glioblastoma in 2009 [12-15]. Despite its approval, multiple large phase 3 clinical trials have since failed to show survival benefit in patients with glioblastoma. This review will focus on the rationale behind anti-angiogenic therapy, data regarding the use of bevacizumab in newly diagnosed and recurrent glioblastoma, mechanisms of resistance to anti-VEGF therapy, and emerging areas of research to define optimal use and maximize clinical benefit.

\section{Angiogenesis}

Tumors acquire blood supply through multiple mechanisms of vessel recruitment: (1) angiogenesis, where new vessels sprout from existing vessels; (2) migration and growth of tumor cells around pre-existing vessels through a process known as vessel co-option; (3) intussusception, or dilation and bifurcation of existing vessels; (4) vascular mimicry, whereby tumor cells incorporate into the endothelial lining; 
Table 1 Landmark clinical trials of antiangiogenic agents for glioblastoma

\begin{tabular}{|c|c|c|c|c|c|c|c|c|}
\hline Trial & Phase & Disease type & Patients (n) & Arms & Median PFS (mo) & PFS-6 (\%) & Median OS (mo) & Reference \\
\hline \multirow[t]{2}{*}{ BRAIN } & \multirow[t]{2}{*}{2} & \multirow[t]{2}{*}{ rGBM } & \multirow[t]{2}{*}{167} & BEV & 4.2 & 42.6 & 9.2 & \multirow[t]{2}{*}{ [3] } \\
\hline & & & & $\mathrm{BEV}$ + irinotecan & 5.6 & 50.3 & 8.7 & \\
\hline NCI & 2 & rGBM & 48 & $\mathrm{BEV}$ & 4.0 & 29.0 & 7.8 & {$[4]$} \\
\hline \multirow[t]{3}{*}{ BELOB } & \multirow[t]{3}{*}{2} & \multirow[t]{3}{*}{ rGBM } & \multirow[t]{3}{*}{153} & BEV & 3.0 & 16.0 & 8.0 & \multirow[t]{3}{*}[5]{} \\
\hline & & & & Lomustine & 1.0 & 13.0 & 8.0 & \\
\hline & & & & $\mathrm{BEV}$ + lomustine & 4.0 & 42.0 & 12.0 & \\
\hline \multirow[t]{2}{*}{ EORTC 26101} & \multirow[t]{2}{*}{3} & \multirow[t]{2}{*}{ rGBM } & \multirow[t]{2}{*}{437} & $\mathrm{BEV}$ + lomustine & 4.2 & NR & 9.1 & \multirow[t]{2}{*}[6]{} \\
\hline & & & & Lomustine & 1.5 & NR & 8.6 & \\
\hline \multirow[t]{3}{*}{ REGAL } & \multirow[t]{3}{*}{3} & \multirow[t]{3}{*}{ rGBM } & \multirow[t]{3}{*}{325} & Cediranib & 92 days & 16.0 & 8.0 & \multirow[t]{3}{*}{ [7] } \\
\hline & & & & $\begin{array}{l}\text { Cediranib + } \\
\text { lomustine }\end{array}$ & 125 days & 35.0 & 9.4 & \\
\hline & & & & Lomustine + placebo & 82 days & 25.0 & 9.8 & \\
\hline \multirow[t]{2}{*}{ Enzastaurin } & \multirow[t]{2}{*}{3} & \multirow[t]{2}{*}{ rGBM } & \multirow[t]{2}{*}{266} & Enzastaurin & 1.5 & 11.1 & 6.6 & \multirow[t]{2}{*}[8]{} \\
\hline & & & & Lomustine & 1.6 & 19 & 7.1 & \\
\hline \multirow[t]{2}{*}{ RTOG 0825} & \multirow[t]{2}{*}{3} & \multirow[t]{2}{*}{ nGBM } & \multirow[t]{2}{*}{637} & $\mathrm{BEV}+\mathrm{TMZ} / \mathrm{XRT}$ & 10.7 & NR & 15.7 & \multirow[t]{2}{*}{ [9] } \\
\hline & & & & TMZ/XRT & 7.3 & NR & 16.1 & \\
\hline \multirow[t]{2}{*}{ AVAGlio } & \multirow[t]{2}{*}{3} & \multirow[t]{2}{*}{ nGBM } & \multirow[t]{2}{*}{921} & $\mathrm{BEV}+\mathrm{TMZ} / \mathrm{XRT}$ & 10.6 & NR & 16.9 & \multirow[t]{2}{*}[10]{} \\
\hline & & & & TMZ/XRT & 6.2 & NR & 16.8 & \\
\hline GLARIUS & 2 & $\begin{array}{l}\text { nGBM (MGMT } \\
\text { unmethylated) }\end{array}$ & 170 & $\begin{array}{l}\mathrm{BEV}+ \\
\quad \text { irinotecan/XRT }\end{array}$ & 9.7 & 71.1 & 16.6 & {$[11]$} \\
\hline & & & & TMZ/XRT & 5.9 & 26.2 & 17.3 & \\
\hline
\end{tabular}

Abbreviations: $r G B M$ recurrent glioblastoma, $n G B M$ newly diagnosed glioblastoma, $B E V$ bevacizumab, $T M Z$ temozolomide, $X R T$ radiation therapy, $N R$ not reported

(5) recruitment of endothelial progenitor cells; and (6) differentiation of cancer stem-like cells into endothelial cells [16-18]. Nonsprouting mechanisms of vessel recruitment may be important for development of resistance to antiangiogenic therapy (Fig. 1).

Glioblastoma vasculature is functionally and structurally abnormal, characterized by uneven vessel diameter, permeability, tortuosity, and thickened basement membranes. This leads to hypoxic regions observed histopathologically as pseudopalisading necrosis, another hallmark of glioblastoma. Hypoxia and angiogenesis are intricately tied to tumor growth and invasion. Hypoxia results in upregulation of hypoxiainducible factor- $1 \alpha$ (HIF-1 $\alpha$ ), which subsequently leads to upregulation of VEGF [20]. It also promotes cancer cell invasion, genetic instability, stem-like phenotype, epithelial to mesenchymal transition, altered metabolism, and creation of an immunosuppressive environment [21]. In addition to VEGF, other pro-angiogenic factors upregulated in glioblastomas include hepatocyte growth factor (HGF), fibroblast growth factor (FGF), platelet-derived growth factor (PDGF), angiopoietins, and interleukin-8 [22-25]. Angiogenesis is also constitutively activated through non-hypoxia dependent pathways such as Ras/mitogen-activated protein kinase (MAPK) and phosphoinositide-3-kinase (PI3K) [26]. The many contributing pathways involved in angiogenesis create multiple opportunities for therapeutic targeting.

\section{Targeting Angiogenesis and VEGF}

Anti-angiogenic strategies have primarily focused on VEGF signaling by using antibodies to bind VEGF, blocking VEGF receptor (VEGFR) activation via small molecule tyrosine kinase inhibitors (TKIs), and directly blocking VEGF binding via engineered peptides or monoclonal antibodies. Many TKIs also have activity against other kinases involved in signaling in endothelial cells and pericytes, such as PDGFR, FGFR, EGFR, KIT, RAF, and RET, and even some types of cancer cells.

Early trials on anti-angiogenic agents in glioblastoma included thalidomide, a weak inhibitor of FGF and VEGFmediated angiogenesis. It demonstrated modest activity when used alone or in combination with carmustine for recurrent glioblastoma, however there was no benefit observed when it was combined with the DNA alkylating agent temozolomide and radiation in the newly diagnosed setting [27-31]. Other potential angiogenesis inhibitors studied with no clear benefit included agents such as the thalidomide analogue 


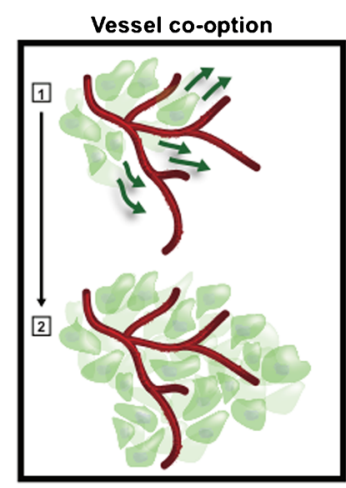

Pericyte-covered tumor vessels
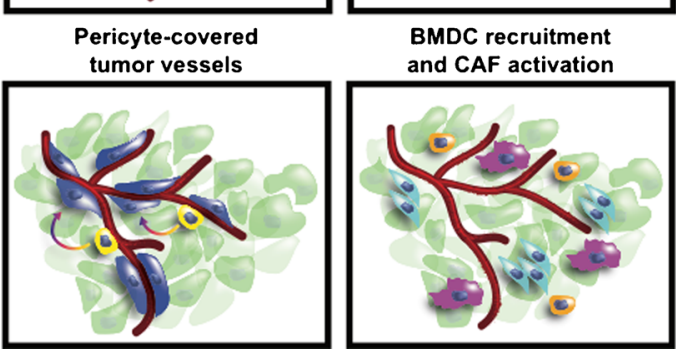

Vascular intussusception

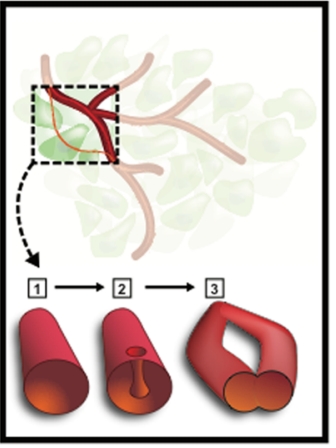

BMDC recruitment and CAF activation

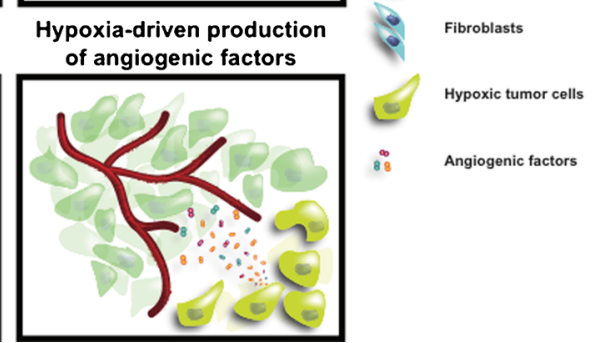

Fig. 1 Mechanisms of angiogenesis and resistance to anti-VEGF therapy. Resistance to anti-VEGF therapy is thought to occur through the following mechanisms: (1) Vessel co-option: tumor cell migration and growth along native vasculature without new blood vessel formation. (2) Vascular intussusception: enlargement and bifurcation of existing vessels. (3) Vascular mimicry: incorporation of tumor cells into the endothelial lining, possibly through endothelial differentiation of tumor stem cells. (4) Peri-cyte covered vessels may be inherently more resistant to VEGF signaling. (5) Recruitment of bone marrow-derived cells and cancer associated fibroblasts which provide paracrine support. (6) Hypoxia-driven release of alternate angiogenic factors. Reprinted with permission from Lu-Emerson et al, J Clin Oncol 2015 [19]

lenalidomide, carboxyamidrotriazole, and penicillamine [32-35].

Recent clinical trials have focused on more potent inhibitors of angiogenesis, particularly bevacizumab, a humanized monoclonal antibody that binds VEGF-A with high affinity and specificity. Bevacizumab inhibits angiogenesis and tumor growth in preclinical models of glioblastoma [36-39]. The initial proposed mechanism of action is through decreased tumor perfusion, thereby depriving the tumor of nutrients and oxygen [40]. However, more recent studies have suggested that in the initial stages of treatment and at low doses, anti-angiogenic agents such as bevacizumab normalize tumor blood vessels, thereby improving vessel function and reducing tumor-associated edema [21]. Figure 2 demonstrates the frequently observed partial response with decrease in $\mathrm{T} 2$ hyperintensity and enhancement seen after bevacizumab initiation. Unfortunately, this usually transient phenomenon, known as pseudoresponse, does not confer a survival benefit [41, 42]. Increased perfusion is also observed in a subset of glioblastoma patients after bevacizumab and may sensitize the tumor to radiation and chemotherapy [43, 44].

Numerous receptor tyrosine kinase inhibitors of VEGF and other pro-angiogenic pathways have been tested in clinical trials. However, with the exception of cediranib and enzastaurin, an oral serine/threonine kinase inhibitor, none have progressed beyond phase 2 clinical trials. Despite data showing promising radiographic response rates and progression free survival (PFS) at 6 months, a phase 3 trial

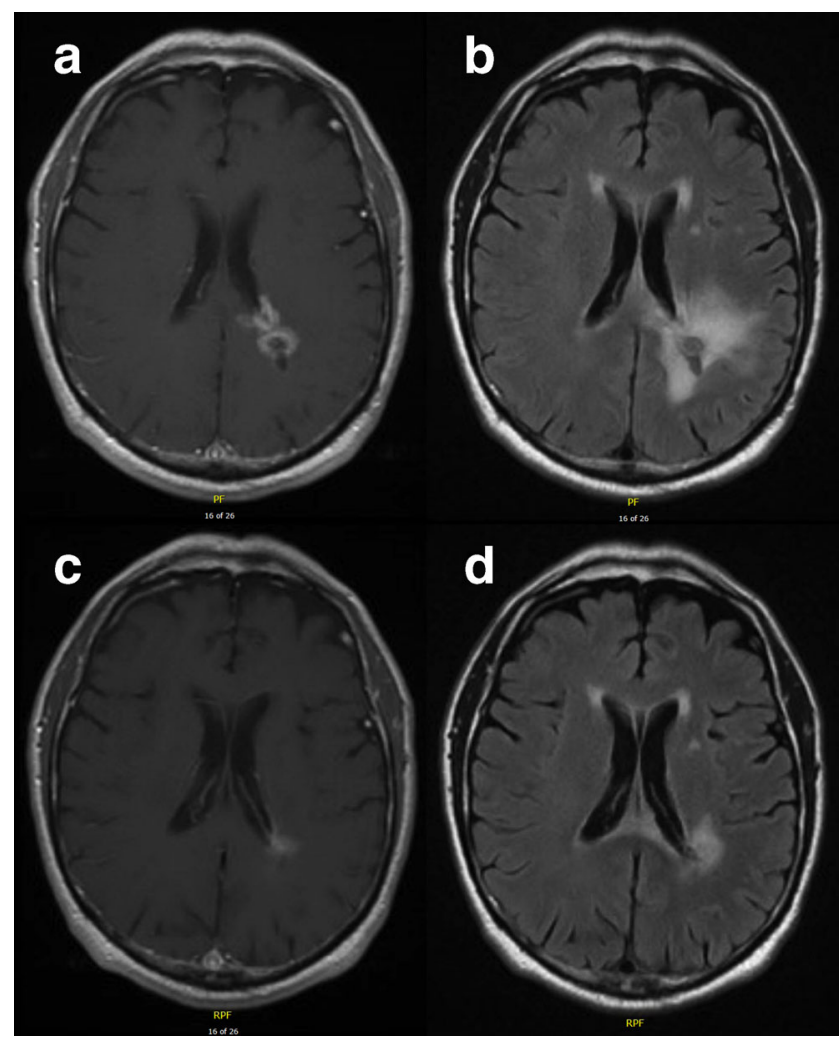

Fig. 2 Radiographic response to bevacizumab. Magnetic resonance imaging of a patient with left parietal glioblastoma before $(A, B)$ and after $(C, D)$ bevacizumab. There is decreased enhancement $(A, C)$ and decreased T2/FLAIR hyperintensity $(B, D)$ two months after initiating treatment 
demonstrated no difference in PFS or overall survival (OS) in recurrent glioblastoma patients treated with cediranib monotherapy or cediranib with lomustine, an alkylating nitrosourea, versus lomustine alone [45, ]. Enzastaurin, which targets the protein kinase $\mathrm{C}$ and PI3K/AKT pathways, showed an objective radiographic response rate of $22 \%$ when combined with bevacizumab in a phase 2 study in patients with recurrent glioblastoma [46]. However, a randomized, phase 3 trial of enzastaurin in recurrent glioblastoma showed no improvement in PFS and OS compared to lomustine [8].

In addition to VEGF, other proangiogenic targets include integrins [47-50], transforming growth factor beta (TGF- $\beta$ ) [51, 52], and matrix metalloproteinases (MMPs) [53-55]. MMPs are important for vascular remodeling, recruitment of bone marrow derived cells, and release of cytokines such as integrin [56, 57]. Integrins mediate cell adhesion, proliferation, migration, and invasion and also play a role in activation of TGF- $\beta$, which additionally is critical for tumor growth, invasion, and immune regulation [58]. The angiopoietin-2 (Ang-2)/TIE2 pathway has been targeted in systemic malignancies and preclinical models of glioblastoma given its interactions with VEGF and role in vessel stabilization as well as recruitment and reprograming of tumor-associated macrophages [59]. Other pathways under investigation include Delta-like ligand 4 (DLL4)/Notch, important for angiogenic sprouting [60, 61]; HGF/c-Met, important for tumor growth and angiogenesis $[62,63]$; and $\mathrm{Wnt} / \beta$-catenin, thought to promote glioblastoma stem cells, cell invasion and migration, and treatment resistance [64].

As bevacizumab has been the best-studied anti-angiogenic agent with the most promising results, the following sections will focus on the data regarding its use for recurrent and newly diagnosed glioblastoma.

\section{Recurrent Glioblastoma}

Two prospective, phase 2 studies led to accelerated FDA approval of bevacizumab as monotherapy for recurrent glioblastoma in 2009. The BRAIN study, completed in 2007, compared bevacizumab to bevacizumab plus irinotecan, an inhibitor of topoisomerase I. Radiographic response was assessed by the WHO Response Evaluation Criteria, which is based on the longest unidimensional measurement of a target enhancing lesion [65], although non-enhancing lesions and steroid dosage were also considered in the study. The overall response rates (ORR) were $28.2 \%$ and $37.8 \%$ with PFS-6 of $42.6 \%$ and $50.3 \%$, respectively [66]. However, the trial was not designed as a superiority trial and allowed for crossover from single agent bevacizumab to the combination arm, potentially confounding the results. A second, single-arm study evaluated 48 recurrent glioblastoma patients treated with bevacizumab at the National Cancer Institute (NCI) and found an ORR of
$35 \%$ and PFS-6 of 29\% [67]. Both the Levin and the MacDonald criteria were used, with the Levin criteria qualitatively assessing enhancement, edema, and mass effect, and the MacDonald criteria focusing on objective bidimensional measurements of an enhancing target lesion [66, 67]. While the FDA approved the use of bevacizumab monotherapy in recurrent GBM based on these two trials, the European Medicines Agency declined approval due to the lack of a nonbevacizumab control arm, modest improvement in OS, and challenges with radiographic response assessment [68].

Multiple studies have also evaluated the use of bevacizumab in combination regimens. The BELOB trial was a randomized phase 2 study of 148 patients with recurrent glioblastoma randomized to lomustine, bevacizumab, or both. Combination therapy resulted in a PFS- 6 of $41 \%$ compared to $11 \%$ and $18 \%$ with OS at 9 months of $59 \%$ compared to $43 \%$ and $38 \%$ for lomustine and bevacizumab alone, respectively [69]. Based on these results, a phase 3 study (EORTC 26101) was conducted to compare lomustine versus lomustine plus bevacizumab. There was no significant difference in OS for combination treatment versus lomustine alone, although median PFS was increased from 1.5 to 4.2 months for combination therapy [70].

Additional phase 2 trials have evaluated bevacizumab in combination with irinotecan, cetuximab, carboplatin, etoposide, fotemustine, sorafenib, temozolomide, erlotinib, panobinostat, and temsirolimus [66, 69-88]. There have also been trials evaluating bevacizumab and re-irradiation [89-91]. Unfortunately, none of these trials have demonstrated outcomes superior to historical controls treated with bevacizumab alone.

\section{Newly Diagnosed Glioblastoma}

Several early, single-arm phase 2 studies evaluating the use of bevacizumab with temozolomide and radiation showed near doubling of median PFS to 13-14 months compared to historical controls. However, only a modest improvement in median OS to 10-21 months was observed [92-94].

Two randomized, placebo-controlled, phase 3 trials investigated the addition of bevacizumab to standard temozolomide plus radiation (chemoradiation) in patients with newly diagnosed glioblastoma. The AVAglio study compared patients randomized to bevacizumab versus placebo in combination with standard chemoradiation. The PFS was significantly prolonged at 10.6 months in the bevacizumab group compared to 6.2 months in the standard therapy group [95]. The RTOG 0825 study also compared bevacizumab to placebo in combination with standard chemoradiation and demonstrated an improvement in PFS to 10.7 months versus 7.3 months with placebo, although this did not meet the predefined significance level of $\mathrm{P}=0.004$ [96]. Unfortunately, both studies failed to demonstrate a benefit in OS. Both studies also had crossover rates of 30 to $50 \%$. Thus, the true impact on OS may 
have been obscured by a large number of patients in the placebo arm who subsequently were treated with bevacizumab at the time of disease progression.

The AVAglio and RTOG 0825 studies also differed in significant ways. While AVAglio used the revised Response Assessment in Neuro-oncology (RANO) criteria to assess disease progression, RTOG 0825 used the traditional Macdonald criteria [67, 95, 96]. Unlike the RANO criteria, the MacDonald criteria do not account for non-enhancing tumor volume, which may be important given the alteration of contrast permeability with bevacizumab. Both trials also attempted to assess other measures of clinical benefit such as performance status, corticosteroid requirement, and quality of life measures. Interestingly, the studies had some divergent findings. While the AVAglio trial showed that bevacizumab prolonged maintenance of performance status, decreased steroid utilization, and prolonged time to deterioration in prespecified cognitive domains, RTOG 0825 found that bevacizumab led to worsened cognitive function. The cause of the differences is unclear, but possible explanations include different radiographic response criteria, substantial dropout in the RTOG trial, and differences in statistical modeling.

Combination therapies with bevacizumab in newly diagnosed glioblastoma have also been assessed. The randomized, phase 2 GLAIRUS study compared standard of care chemoradiation with temozolomide versus radiation with bevacizumab and irinotecan in patients whose tumors expressed the DNA repair enzyme O6-methyl guanine DNA methyltransferase (MGMT). Loss of MGMT function through gene promoter methylation has been shown to confer increased sensitivity to therapy with temozolomide in glioblastoma [97]. The GLARIUS trial found that PFS was significantly prolonged at 9.7 months in the bevacizumab plus irinotecan arm compared to 5.99 months in the control arm. However, the OS did not significantly differ with OS of 17.5 months in the control arm compared to 16.6 months in the experimental arm. Neither therapy regimen was superior in delaying the time to deterioration in pre-specified dimensions of quality of life [11].

\section{Mechanisms of Resistance}

Despite impressive radiographic responses and improved PFS, response to anti-angiogenic therapy is unfortunately not durable. Alternative mechanisms of vessel recruitment are ultimately utilized (Fig. 1). Local hypoxia may trigger alternative pro-angiogenic factors such as HGF, FGF, Ang-2, SDF1 $\alpha$, and interleukin- 8 [98-102]. Preclinical studies have shown that dual targeting of VEGF and Ang-2 may overcome this form of resistance to anti-VEGF monotherapy [103-105]. Vessel co-option, the process whereby tumors utilize native brain vessels to recruit blood supply, is also under investigation as an escape mechanism to anti-angiogenic therapy [21].
The molecular mechanisms of vessel co-option are poorly understood and may yield novel therapeutic approaches once the pathways are identified.

Some tumor vessel subtypes are thought to have inherent insensitivity to VEGF inhibition due to decreased sensitivity of pericytes [106, 107]. There is also preclinical data that antiangiogenic therapy induces transformation from a proneural to a more invasive mesenchymal phenotype, including upregulation and increased phosphorylation of the receptor tyrosine kinase c-Met [108-110]. Increased invasion may also be mediated by MMPs [111]. Retrospective data in patients found that treatment with bevacizumab was associated with non-enhancing, diffuse or distant recurrence (Fig. 3) [70].

Given the potential disease progression via non-enhancing, infiltrative or invasive disease with anti-angiogenic therapy, combination therapy with an agent that targets invasion may also be a promising strategy to overcome resistance. For example, inhibition and knockdown of c-Met inhibit tumor growth and prolong survival in GBM mouse models [108-110]. Interim analysis from a completed phase 2 trial of the monovalent MET inhibitor onartuzumab or placebo with bevacizumab showed no difference in PFS, OS, or

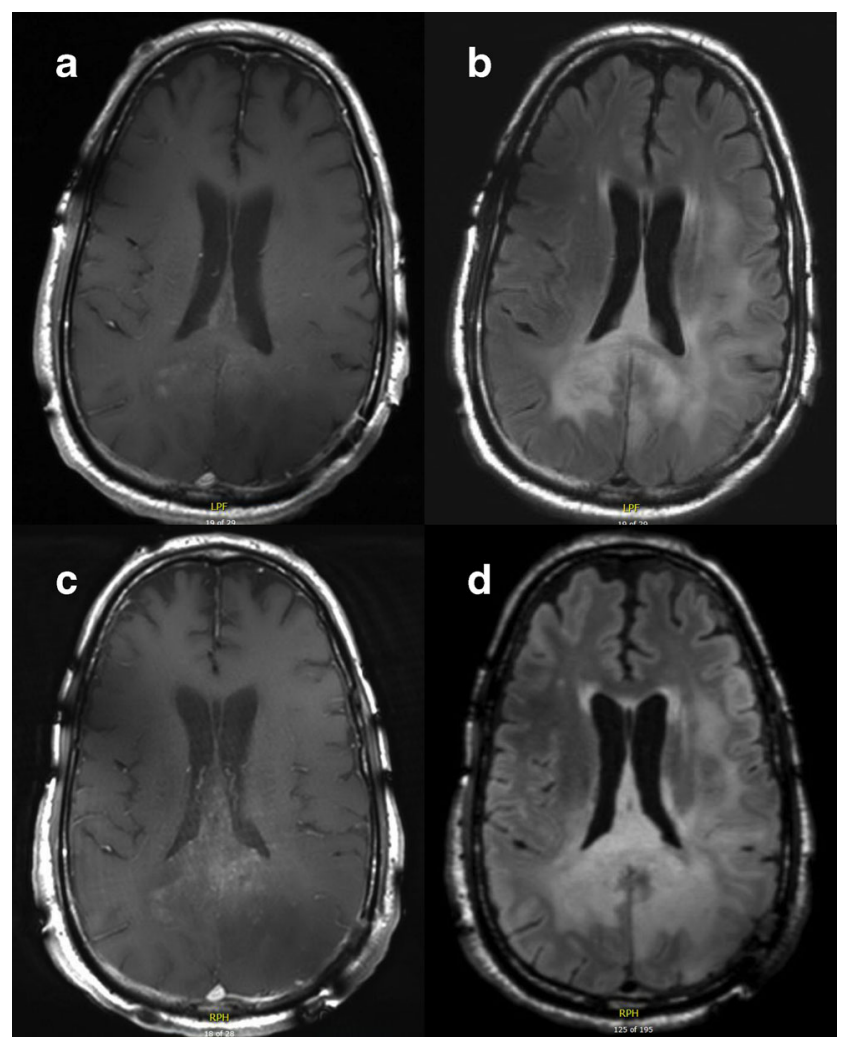

Fig 3 Disease progression on bevacizumab. Magnetic resonance imaging from a patient with glioblastoma who progressed on bevacizumab. Panels $A$ and $B$ show T1 post-contrast and T2/FLAIR sequences, respectively, of a patient with glioblastoma involving the splenium of the corpus callosum. Panels $C$ and $D$ show post-contrast and T2/FLAIR sequences after one month with notable increase in patchy enhancement and corresponding increase in $\mathrm{T} 2$ hyperintensity 
ORR, however a phase 1 trial of another c-Met inhibitor INC280 is ongoing (NCT02386826).

\section{Anti-angiogenesis and the Immune System}

Angiogenesis plays an important role in tumor immunity, and the immune composition of the tumor microenvironment changes with vascular normalization and decreased local hypoxia. Preclinical data in extracranial tumors suggest that antiangiogenic therapies increase tumor delivery of activated $\mathrm{T}$ cells, rendering the tumor more susceptible to immune attack [112]. Anti-angiogenic therapy also facilitates the recruitment of bone marrow-derived cells and polarization of tumor associated macrophages to immune stimulatory M1-skewed macrophages (Fig. 4) [20, 113, 114]. Furthermore, increased circulating levels of VEGF inhibit T cell immune response by suppressing maturation of dendritic cell precursors and promoting proliferation of regulatory T cells [112]. However, studies have also suggested that immune activation may play a role in resistance to anti-angiogenic therapy. Peripheral mobilization of myeloid cells via granulocyte colony stimulating factor (G-CSF) has been associated with refractoriness to anti-angiogenic therapy $[114,115]$. Pro-angiogenic M2-polarized macrophages may also be important, and strategies to reprogram or inhibit the M2 phenotype, such as through inhibition of Ang-2 or proinflammatory cytokines, have been shown to augment anti-angiogenic therapy in animal models [103, 116, 117].

Despite the conventional notion that the central nervous system is immune-privileged due to the blood brain barrier, the immunomodulatory effect of anti-VEGF has led to recent clinical trials combining bevacizumab with immunotherapy in hopes of a synergistic effect in facilitating anti-tumor immunity. Preliminary results from a phase 2 trial of standard of care chemoradiation versus standard of care plus the dendritic cell vaccine AV0113 in patients with newly diagnosed glioblastoma showed no difference in PFS or OS; however, in the subgroup of 22 patients that received the vaccine as second-line therapy with bevacizumab, there was an improvement in OS compared to the control arm $(535 \pm 155$ days versus $406 \pm 224$ days $)$ [118]. Preliminary results from the phase 2 study of patients with EGFRvIII mutant recurrent glioblastomas (approximately $20-30 \%$ of all primary glioblastomas) demonstrate that the combination of rindopepimut, a peptide vaccine against EGFRvIII, with bevacizumab prolonged median OS from 8.8 months in the control arm (bevacizumab plus keyhole limpet hemocyanin) to 12 months in the experimental arm [54]. PFS-6 was also significantly increased from $11 \%$ to $27 \%$. However, the phase 3 ACT IV trial of standard of care with temozolomide versus temozolomide plus rindopepimut was recently stopped after interim analysis showed no difference in median OS (20.4 months in the rindopepimut group versus 21.1 months in the control group, HR 0.99) [119]. The question remains whether rindopepimut may have a more robust effect on survival when combined with an anti-angiogenic therapy such as bevacizumab. In addition to vaccine strategies, adoptive cell transfer (ACT) combined with anti-angiogenic therapy in a mouse model of B16 melanoma showed a synergistic effect with increased infiltration of transferred cells and prolonged survival compared to ACT alone [120].

Ongoing clinical trials for glioblastoma patients are evaluating the use of anti-angiogenic therapy in combination with immune checkpoint inhibitors (pembrolizumab NCT02337491 and durvalumab NCT02336165) and vaccines (SL-701 NCT02078648 and heat shock protein peptide complexes NCT01814813).

\section{Searching for Biomarkers}

Unlike other targeted therapies, no established biomarkers currently exist to help predict response to anti-angiogenic

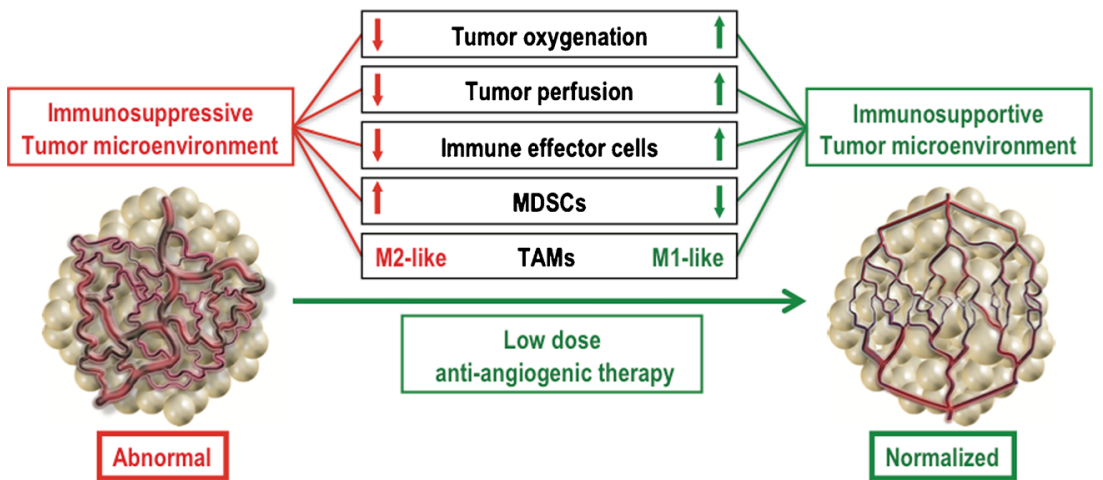

Fig. 4 Anti-angiogenic therapy and the immune microenvironment. Abnormal tumor vasculature creates a hypoxic tumor microenvironment, thereby decreasing tumor oxygenation, tumor perfusion, reducing the number of immune effector cells in the microenvironment, increasing myeloid-derived stem cells, and polarizing tumor associated macrophages to the immune inhibitory M2like phenotype. Low dose anti-VEGF therapy is thought to normalize blood vessels and have the opposite effect. Reprinted with permission from Huang et al, Cancer Res [112] 
therapy. Tissue, serologic, and imaging markers are all under active investigation, although none have yet been validated for use in clinical practice.

\section{Tumor Tissue}

Tumor tissue biomarkers have been evaluated mostly in the setting of bevacizumab. In the RTOG-0825 randomized trial, a 10-gene panel assessed the degree of mesenchymal gene enrichment and was significantly associated with worse PFS and OS in response to bevacizumab [121]. Other tissue markers include levels of VEGF, carbonic anhydrase 9 (a marker of hypoxia), and the number of CD68+, CD11+ tumor-associated macrophages [122, 123]. Markers that have not been found to predict response include MGMT methylation status, IDH1, EGFR, PDGFR- $\alpha$, and c-KIT [124-126].

\section{Blood Biomarkers}

Circulating blood biomarkers are particularly important for glioblastomas as repeat surgery for tissue sampling is often not feasible. Candidate biomarkers include VEGF and sVEGFR2, SDF-1 $\alpha$, PIGF, and MMPs [95, 113, 127-129]. Some studies have shown associations between changes in biomarker levels and outcomes. For example, elevated levels of MMP-9, sVEGFR-1 (a negative regulator of VEGF), and SDF- $1 \alpha$ have been associated with decreased survival in patients receiving anti-angiogenic agents such as cediranib and aflibercept $[45,130]$. On the other hand, increased collagen IV levels have been associated with improved PFS in recurrent glioblastoma [131]. The identification of validated serum biomarkers will aid in drug development as well as noninvasive monitoring and treatment selection in patients.

\section{Imaging Biomarkers}

In 2010, the Response Assessment in Neuro-Oncology Working Group proposed the RANO criteria to account for rapid reduction of contrast enhancement after anti-angiogenic therapy as well as transient increase in tumor enhancement shortly after chemoradiation (pseudoprogression) [95]. The criteria include the assessment of non-enhancing lesions and also offers guidelines for distinguishing pseudoprogression from progression. Quantifiable radiographic changes after treatment have led to investigation of various imaging markers to help predict tumor response, including apparent diffusion coefficient [132], restriction spectrum imaging [133], dynamic contrast enhanced (DCE) and dynamic susceptibility-contrast (DSC) techniques [134, 135], vessel architectural imaging [136], and dopamine and positron emission tomography $[137,138]$. Consistent with the vascular normalization hypothesis, data suggest that improved perfusion is correlated with increased OS in both newly-diagnosed and recurrent glioblastoma patients treated with the pan-VEGF inhibitor cediranib. In one study, patients with recurrent glioblastoma treated with cediranib, an oral pan-VEGF inhibitor, who had sustained increase in perfusion longer than one month had increased OS of 348 days compared to patients with decreased or stable tumor perfusion (213 days and 169 days, respectively, P < 0.01) [139]. Another study of cediranib in newly diagnosed glioblastoma patients found that patients with durably increased perfusion had a mean OS of 26.3 months compared to 17 months in those with stable or decreased perfusion $(\mathrm{P}<$ $0.05)$ [126]. Thus, early imaging changes in response to antiangiogenic therapy may help identify patients more likely to benefit from anti-VEGF therapy.

\section{Future Directions}

While bevacizumab continues to be a mainstay in the treatment of recurrent glioblastoma, the lack of survival benefit in clinical trials has prompted investigation of biomarkers that can help determine the optimum patient population and predict radiographic response. Although the RANO criteria does account for nonenhancing disease and decreased enhancement after anti-angiogenic therapy, more research is needed to distinguish treatment effect from disease progression and further clarify radiographic response patterns in the setting of altered vascular permeability.

The lack of survival benefit despite increase in progression free survival also highlights the potential importance of secondary endpoints such as quality of life measures and steroid usage. Given the vital location, slight radiographic progression of a glioblastoma or increased edema can translate into a disproportionate impact on functional status. While clinical trials and new therapies should continue to focus on prolonging survival, there remains a need to better characterize the impact on day-to-day challenges patients face.

Another area of investigation includes optimal dosing and timing of bevacizumab or other anti-angiogenic therapies. Retrospective data suggest that the treatment of patients with high-grade glioma with low doses of bevacizumab $(5 \mathrm{mg} / \mathrm{kg}$ per week or $7.5 \mathrm{mg} / \mathrm{kg}$ every 3-4 weeks) may be superior to standard dosing, potentially due to vascular normalization at lower doses [140,141]. A recent randomized phase 2 trial comparing low dose bevacizumab plus lomustine to standard dose bevacizumab monotherapy in recurrent GBM showed no difference in the primary endpoint of PFS (4.34 months for the combination arm versus 4.11 months for bevacizumab monotherapy, $p=0.19$ ) [142]. However, there was a trend toward improved median PFS in patients with first recurrence. Due to the potential of rebound cerebral edema after discontinuation of bevacizumab, salvage therapy has been difficult, leading many patients and oncologists to delay bevacizumab as long as possible. However, it is unclear to what extent this delay is 
warranted as additional retrospective data in systemic malignancies indicate that there may be benefit to continuing antiangiogenic therapy past progression [143-146].

While optimizing bevacizumab administration is important, the multiple, complex pathways that promote angiogenesis also support the use of combination strategies. Improved orthotopic animal models from patient-derived tumors will play an important role in the evaluation of new therapies, treatment combinations, and resistance mechanisms, although these models will have limited utility in the evaluation of immunotherapies. It is notable that the only randomized clinical trial that appeared to confer some improvement in survival was in patients with recurrent glioblastoma treated with bevacizumab and chemotherapy. Thus, targeting several pathways or combining anti-angiogenic agents with other classes of drugs such as immunotherapy may prevent the development of treatment resistance and maximize survival benefit.

Required Author Forms Disclosure forms provided by the authors are available with the online version of this article.

\section{References}

1. Stupp R, Mason WP, van den Bent MJ, et al (2005) Radiotherapy plus concomitant and adjuvant temozolomide for glioblastoma. N Engl J Med 352:987-996

2. Gilbert MR, Wang M, Aldape KD, et al (2013) Dose-dense temozolomide for newly diagnosed glioblastoma: a randomized phase III clinical trial. J Clin Oncol 31:4085-91

3. Friedman HS, Prados MD, Wen PY, et al (2009) Bevacizumab alone and in combination with irinotecan in recurrent glioblastoma. J Clin Oncol 27:4733-4740

4. Kreisl TN, Kim L, Moore K, et al (2009) Phase II trial of singleagent bevacizumab followed by bevacizumab plus irinotecan at tumor progression in recurrent glioblastoma. J Clin Oncol 27:740 745

5. Taal W, Oosterkamp HM, Walenkamp AME, et al (2014) Singleagent bevacizumab or lomustine versus a combination of bevacizumab plus lomustine in patients with recurrent glioblastoma (BELOB trial): A randomised controlled phase 2 trial. Lancet Oncol 15:943-953

6. Wick W, Brandes A, Gorlia T, et al (2015) Phase III trial exploring the combination of bevacizumab and lomustine in patients with first recurrence of a glioblastoma: the EORTC 26101 trial. Neuro Oncol 17:v1.5-v1

7. Batchelor TT, Mulholland P, Neyns B, et al (2013) Phase III randomized trial comparing the efficacy of cediranib as monotherapy, and in combination with lomustine, versus lomustine alone in patients with recurrent glioblastoma. J Clin Oncol 31:3212-3218

8. Wick W, Puduvalli VK, Chamberlain MC, et al (2010) Phase III study of enzastaurin compared with lomustine in the treatment of recurrent intracranial glioblastoma. J Clin Oncol 28:1168-1174

9. Gilbert MR, Dignam JJ, Armstrong TS, et al (2014) A randomized trial of bevacizumab for newly diagnosed glioblastoma. N Engl J Med 370:699-708

10. Chinot OL, Wick W, Mason W, et al (2014) Bevacizumab plus radiotherapy-temozolomide for newly diagnosed glioblastoma. $\mathrm{N}$ Engl J Med 370:709-722
11. Herrlinger U, Schäfer N, Steinbach JP, et al (2016) Bevacizumab plus irinotecan versus temozolomide in newly diagnosed O6methylguanine-DNA methyltransferase nonmethylated glioblastoma: the randomized GLARIUS trial. J Clin Oncol. doi: 10.1200/JCO.2015.63.4691

12. Hurwitz H, Fehrenbacher L, Novotny W, et al (2004) Bevacizumab plus irinotecan, fluorouracil, and leucovorin for metastatic colorectal cancer. N Engl J Med 350:2335-42

13. Cohen MH, Gootenberg J, Keegan P, Pazdur R (2007) FDA drug approval summary: bevacizumab (Avastin(R)) plus carboplatin and paclitaxel as first-line treatment of advanced/metastatic recurrent nonsquamous non-small cell lung cancer. Oncologist 12:713718

14. Summers J, Cohen MH, Keegan P, Pazdur R (2010) FDA drug approval summary: bevacizumab plus interferon for advanced renal cell carcinoma. Oncologist 15:104-111

15. Cohen MH, Shen YL, Keegan P, Pazdur R (2009) FDA drug approval summary: bevacizumab (Avastin) as treatment of recurrent glioblastoma multiforme. Oncologist 14:1131-1138

16. Carmeliet P, Jain RK (2011) Molecular mechanisms and clinical applications of angiogenesis. Nature 473:298-307

17. Wang R, Chadalavada K, Wilshire J, Kowalik U, Hovinga KE, Geber A, Fligelman B, Leversha M, Brennan C, Tabar V (2010) Glioblastoma stem-like cells give rise to tumour endothelium. Nature 468:829-833

18. Ricci-Vitiani L, Pallini R, Biffoni M, et al (2010) Tumour vascularization via endothelial differentiation of glioblastoma stem-like cells. Nature 468:824-8

19. Lu-Emerson C, Duda DG, Emblem KE, Taylor JW, Gerstner ER, Loeffler JS, Batchelor TT, Jain RK (2015) Lessons from antivascular endothelial growth factor and anti-vascular endothelial growth factor receptor trials in patients with glioblastoma. J Clin Oncol 33:1197-1213

20. Du R, Lu K V, Petritsch C, et al (2008) HIF1alpha induces the recruitment of bone marrow-derived vascular modulatory cells to regulate tumor angiogenesis and invasion. Cancer Cell 13:206-20

21. Jain RK (2014) Antiangiogenesis strategies revisited: from starving tumors to alleviating hypoxia. Cancer Cell 26:605-622

22. Shih AH, Holland EC (2006) Platelet-derived growth factor (PDGF) and glial tumorigenesis. Cancer Lett 232:139-147

23. Reiss Y, Machein MR, Plate KH (2005) The role of angiopoietins during angiogenesis in gliomas. Brain Pathol 15:311-317

24. Brat DJ, Bellail AC, Van Meir EG (2005) The role of interleukin-8 and its receptors in gliomagenesis and tumoral angiogenesis. Neuro Oncol 7:122-33

25. Schmidt NO, Westphal M, Hagel C, Ergün S, Stavrou D, Rosen EM, Lamszus K (1999) Levels of vascular endothelial growth factor, hepatocyte growth factor/scatter factor and basic fibroblast growth factor in human gliomas and their relation to angiogenesis. Int J Cancer 84:10-8

26. Jayson GC, Kerbel R, Ellis LM, Harris AL (2016) Antiangiogenic therapy in oncology: current status and future directions. Lancet (London, England) 70:6171-80

27. Fine HA, Figg WD, Jaeckle K, et al (2000) Phase II trial of the antiangiogenic agent thalidomide in patients with recurrent highgrade gliomas. J Clin Oncol 18:708-15

28. Marx GM, Pavlakis N, McCowatt S, Boyle FM, Levi JA, Bell DR, Cook R, Biggs M, Little N, Wheeler HR (2001) Phase II study of thalidomide in the treatment of recurrent glioblastoma multiforme. J Neurooncol 54:31-8

29. Chang SM, Lamborn KR, Malec M, Larson D, Wara W, Sneed P, Rabbitt J, Page M, Nicholas MK, Prados MD (2004) Phase II study of temozolomide and thalidomide with radiation therapy for newly diagnosed glioblastoma multiforme. Int J Radiat Oncol Biol Phys 60:353-357 
30. Fine HA, Wen PY, Maher EA, Viscosi E, Batchelor T, Lakhani N, Figg WD, Purow BW, Borkowf CB (2003) Phase II trial of thalidomide and carmustine for patients with recurrent high-grade gliomas. J Clin Oncol 21:2299-2304

31. Kesari S, Schiff D, Henson JW, et al (2008) Phase II study of temozolomide, thalidomide, and celecoxib for newly diagnosed glioblastoma in adults. Neuro Oncol 10:300-308

32. Drappatz J, Wong ET, Schiff D, et al (2009) A pilot safety study of lenalidomide and radiotherapy for patients with newly diagnosed glioblastoma multiforme. Int J Radiat Oncol Biol Phys 73:222227

33. Fine HA, Kim L, Albert PS, Duic JP, Ma H, Zhang W, Tohnya T, Figg WD, Royce C (2007) A phase I trial of lenalidomide in patients with recurrent primary central nervous system tumors. Clin Cancer Res 13:7101-6

34. Mikkelsen T, Lush R, Grossman SA, Carson KA, Fisher JD, Alavi JB, Rosenfeld S (2007) Phase II clinical and pharmacologic study of radiation therapy and carboxyamido-triazole (CAI) in adults with newly diagnosed glioblastoma multiforme. Invest New Drugs 25:259-63

35. Brem S, Grossman S a, Carson K a, New P, Phuphanich S, Alavi JB, Mikkelsen T, Fisher JD (2005) Phase 2 trial of copper depletion and penicillamine as antiangiogenesis therapy of glioblastoma. Neuro Oncol 7:246-253

36. Kim KJ, Li B, Winer J, Armanini M, Gillett N, Phillips HS, Ferrara N (1993) Inhibition of vascular endothelial growth factor-induced angiogenesis suppresses tumour growth in vivo. Nature 362:841-4

37. Rubenstein JL, Kim J, Ozawa T, Zhang M, Westphal M, Deen DF, Shuman MA (2000) Anti-VEGF antibody treatment of glioblastoma prolongs survival but results in increased vascular cooption. Neoplasia 2:306-14

38. Jahnke K, Muldoon LL, Varallyay CG, Lewin SJ, Kraemer DF, Neuwelt EA (2009) Bevacizumab and carboplatin increase survival and asymptomatic tumor volume in a glioma model. Neuro Oncol 11:142-150

39. Lee CG, Heijn M, di Tomaso E, et al (2000) Anti-vascular endothelial growth factor treatment augments tumor radiation response under normoxic or hypoxic conditions. Cancer Res 60:5565-5570

40. Folkman J (1971) Tumor angiogenesis: therapeutic implications. N Engl J Med 285:1182-6

41. Ellingson BM, Cloughesy TF, Lai A, Nghiemphu PL, Mischel PS, Pope WB (2011) Quantitative volumetric analysis of conventional MRI response in recurrent glioblastoma treated with bevacizumab. Neuro Oncol 13:401-409

42. Gorlia T, Stupp R, Brandes AA, et al (2012) New prognostic factors and calculators for outcome prediction in patients with recurrent glioblastoma: A pooled analysis of EORTC Brain Tumour Group phase i and II clinical trials. Eur J Cancer 48: 1176-1184

43. Winkler F, Kozin S V., Tong RT, et al (2004) Kinetics of vascular normalization by VEGFR2 blockade governs brain tumor response to radiation: Role of oxygenation, angiopoietin-1, and matrix metalloproteinases. Cancer Cell 6:553-563

44. Batchelor TT, Sorensen AG, di Tomaso E, et al (2007) AZD2171, a pan-VEGF receptor tyrosine kinase inhibitor, normalizes tumor vasculature and alleviates edema in glioblastoma patients. Cancer Cell 11:83-95

45. Batchelor TT, Duda DG, Di Tomaso E, et al (2010) Phase II study of cediranib, an oral pan-vascular endothelial growth factor receptor tyrosine kinase inhibitor, in patients with recurrent glioblastoma. J Clin Oncol 28:2817-2823

46. Odia Y, Iwamoto FM, Moustakas A, Fraum TJ, Salgado CA, Li A, Kreisl TN, Sul J, Butman JA, Fine HA (2016) A phase II trial of enzastaurin (LY317615) in combination with bevacizumab in adults with recurrent malignant gliomas. J Neurooncol 127:127135

47. Nabors LB, Mikkelsen T, Rosenfeld SS, et al (2007) Phase I and correlative biology study of cilengitide in patients with recurrent malignant glioma. J Clin Oncol 25:1651-7

48. Reardon DA, Fink KL, Mikkelsen T, et al (2008) Randomized phase II study of cilengitide, an integrin-targeting arginineglycine-aspartic acid peptide, in recurrent glioblastoma multiforme. J Clin Oncol 26:5610-5617

49. Stupp R, Hegi ME, Neyns B, et al (2010) Phase I/IIa study of cilengitide and temozolomide with concomitant radiotherapy followed by cilengitide and temozolomide maintenance therapy in patients with newly diagnosed glioblastoma. J Clin Oncol 28: $2712-2718$

50. Stupp R, Hegi ME, Gorlia T, et al (2014) Cilengitide combined with standard treatment for patients with newly diagnosed glioblastoma with methylated MGMT promoter (CENTRIC EORTC 26071-22072 study): a multicentre, randomised, open-label, phase 3 trial. Lancet Oncol 15:1100-1108

51. Bogdahn U, Hau P, Stockhammer G, et al (2011) Targeted therapy for high-grade glioma with the TGF-beta2 inhibitor trabedersen: Results of a randomized and controlled phase IIb study. Neuro Oncol 13:132-142

52. Schlingensiepen KH, Schlingensiepen R, Steinbrecher A, Hau P, Bogdahn U, Fischer-Blass B, Jachimczak P (2006) Targeted tumor therapy with the TGF-beta 2 antisense compound AP 12009. Cytokine Growth Factor Rev 17:129-139

53. Groves MD, Puduvalli VK, Hess KR, Jaeckle KA, Peterson P, Yung WKA, Levin VA (2002) Phase II trial of temozolomide plus the matrix metalloproteinase inhibitor, marimastat, in recurrent and progressive glioblastoma multiforme. J Clin Oncol 20:1383-8

54. Levin VA, Phuphanich S, Glantz MJ, et al (2002) Randomized phase II study of temozolomide (TMZ) with and without the matrix metalloprotease (MMP) inhibitor prinomastat in patients (pts) with glioblastoma multiforme (GBM) following best surgery and radiation therapy. Proc Am Soc Clin Oncol 21:26a

55. Levin VA, Phuphanich S, Yung WKA, Forsyth PA, Del Maestro R, Perry JR, Fuller GN, Baillet M (2006) Randomized, doubleblind, placebo-controlled trial of marimastat in glioblastoma multiforme patients following surgery and irradiation. $\mathrm{J}$ Neurooncol 78:295-302

56. Heissig B, Hattori K, Dias S, et al (2002) Recruitment of stem and progenitor cells from the bone marrow niche requires MMP-9 mediated release of kit-ligand. Cell 109:625-37

57. Rundhaug JE (2005) Matrix metalloproteinases and angiogenesis. Angiogenesis Review Series. J Cell Mol Med 9:267-285

58. Massagué J (2008) TGFb in cancer. Cell 134:215-230

59. Gerald D, Chintharlapalli S, Augustin HG, Benjamin LE (2013) Angiopoietin-2: An attractive target for improved antiangiogenic tumor therapy. Cancer Res 73:1649-1657

60. Kangsamaksin T, Murtomaki A, Kofler NM, Cuervo H, Chaudhri RA, Tattersall IW, Rosenstiel PE, Shawber CJ, Kitajewski J (2015) NOTCH decoys that selectively block DLL/NOTCH or JAG/NOTCH disrupt angiogenesis by unique mechanisms to inhibit tumor growth. Cancer Discov 5:182-97

61. Smith DC, Eisenberg PD, Manikhas G, Chugh R, Gubens MA, Stagg RJ, Kapoun AM, Xu L, Dupont J, Sikic B (2014) A phase I dose escalation and expansion study of the anticancer stem cell agent demcizumab (Anti-DLL4) in patients with previously treated solid tumors. Clin Cancer Res 20:6295-6303

62. Wen PY, Schiff D, Cloughesy TF, et al (2011) A phase II study evaluating the efficacy and safety of AMG 102 (rilotumumab) in patients with recurrent glioblastoma. Neuro Oncol 13:437-446

63. Cloughesy TF, Finocchiaro G, Belda C, Recht L, Brandes AA, Weller M, Liu B, Bruey J, Verret W (2015) Onartuzumab plus bevacizumab versus placebo plus bevacizumab in recurrent 
glioblastoma (GBM): HGF and MGMT biomarker data. J Clin Oncol 33:abstr 2015

64. Lee Y, Lee J-K, Ahn SH, Lee J, Nam D-H (2015) WNT signaling in glioblastoma and therapeutic opportunities. Lab Investig 0:1-14

65. Therasse P, Arbuck SG, Eisenhauer EA, et al (2000) New guidelines to evaluate the response to treatment in solid tumors. J Natl Cancer Inst 92:205-216

66. Levin VA, Crafts DC, Norman DM, Hoffer PB, Spire J-P, Wilson CB (1977) Criteria for evaluating patients undergoing chemotherapy for malignant brain tumors. J Neurosurg 47:329-335

67. Macdonald DR, Cascino TL, Schold SC, Cairncross JG (1990) Response criteria for phase II studies of supratentorial malignant glioma. J Clin Oncol 8:1277-80

68. Wick W, Weller M, Van Den Bent M, Stupp R (2010) Bevacizumab and recurrent malignant gliomas: A European perspective. J Clin Oncol 28:188-189

69. Lee EQ, Reardon DA, Schiff D, et al (2015) Phase II study of panobinostat in combination with bevacizumab for recurrent glioblastoma and anaplastic glioma. Neuro Oncol 17:862-867

70. Norden AD, Young GS, Setayesh K, et al (2008) Bevacizumab for recurrent malignant gliomas: efficacy, toxicity, and patterns of recurrence. Neurology 70:779-87

71. Francesconi AB, Dupre S, Matos M, Martin D, Hughes BG, Wyld DK, Lickliter JD (2010) Carboplatin and etoposide combined with bevacizumab for the treatment of recurrent glioblastoma multiforme. J Clin Neurosci 17:970-4

72. Reardon DA, Desjardins A, Peters KB, et al (2012) Phase II study of carboplatin, irinotecan, and bevacizumab for bevacizumab naïve, recurrent glioblastoma. J Neurooncol 107:155-64

73. Reardon DA, Desjardins A, Vredenburgh JJ, et al (2009) Metronomic chemotherapy with daily, oral etoposide plus bevacizumab for recurrent malignant glioma: a phase II study. Br J Cancer 101:1986-94

74. Ali SA, McHayleh WM, Ahmad A, Sehgal R, Braffet M, Rahman M, Bejjani G, Friedland DM (2008) Bevacizumab and irinotecan therapy in glioblastoma multiforme: a series of 13 cases. J Neurosurg 109:268-72

75. Bokstein F, Shpigel S, Blumenthal DT (2008) Treatment with bevacizumab and irinotecan for recurrent high-grade glial tumors. Cancer 112:2267-2273

76. Kang TY, Jin T, Elinzano H, Peereboom D (2008) Irinotecan and bevacizumab in progressive primary brain tumors, an evaluation of efficacy and safety. J Neurooncol 89:113-118

77. Zuniga RM, Torcuator R, Jain R, Anderson J, Doyle T, Ellika S, Schultz L, Mikkelsen T (2009) Efficacy, safety and patterns of response and recurrence in patients with recurrent high-grade gliomas treated with bevacizumab plus irinotecan. J Neurooncol 91: 329-336

78. Nghiemphu PL, Liu W, Lee Y, et al (2009) Bevacizumab and chemotherapy for recurrent glioblastoma: A single-institution experience. Neurology 72:1217-1222

79. Desjardins A, Reardon DA, Coan A, Marcello J, Herndon JE, Bailey L, Peters KB, Friedman HS, Vredenburgh JJ (2012) Bevacizumab and daily temozolomide for recurrent glioblastoma. Cancer 118:1302-1312

80. Sathornsumetee S, Desjardins A, Vredenburgh JJ, et al (2010) Phase II trial of bevacizumab and erlotinib in patients with recurrent malignant glioma. Neuro Oncol 12:1300-1310

81. Galanis E, Anderson SK, Lafky JM, et al (2013) Phase II study of bevacizumab in combination with sorafenib in recurrent glioblastoma (N0776): a north central cancer treatment group trial. Clin Cancer Res 19:4816-23

82. Drappatz J, Lee EQ, Hammond S, et al (2012) Phase I study of panobinostat in combination with bevacizumab for recurrent highgrade glioma. J Neurooncol 107:133-8
83. Lassen U, Sorensen M, Gaziel TB, Hasselbalch B, Poulsen HS (2013) Phase II study of bevacizumab and temsirolimus combination therapy for recurrent glioblastoma multiforme. Anticancer Res 33:1657-1660

84. Møller S, Grunnet K, Hansen S, Schultz H, Holmberg M, Sorensen M, Poulsen HS, Lassen U (2012) A phase II trial with bevacizumab and irinotecan for patients with primary brain tumors and progression after standard therapy. Acta Oncol (Madr) 51:797-804

85. Soffietti R, Trevisan E, Bertero L, et al (2014) Bevacizumab and fotemustine for recurrent glioblastoma: A phase II study of AINO (Italian Association of Neuro-Oncology). J Neurooncol 116:533541

86. Reardon DA, Desjardins A, Peters KB, et al (2011) Phase 2 study of carboplatin, irinotecan, and bevacizumab for recurrent glioblastoma after progression on bevacizumab therapy. Cancer 117 : 5351-8

87. Raizer JJ, Giglio P, Hu J, et al (2016) A phase II study of bevacizumab and erlotinib after radiation and temozolomide in MGMT unmethylated GBM patients. J Neurooncol 126:185-192

88. Field KM, Simes J, Nowak AK, et al (2015) Randomized phase 2 study of carboplatin and bevacizumab in recurrent glioblastoma. Neuro Oncol 17:1504-1513

89. Cuneo KC, Vredenburgh JJ, Sampson JH, Reardon DA, Desjardins A, Peters KB, Friedman HS, Willett CG, Kirkpatrick JP (2013) Safety and efficacy of stereotactic radiosurgery and adjuvant bevacizumab in patients with recurrent malignant gliomas. Int J Radiat Oncol Biol Phys 82:2018-2024

90. Cabrera AR, Cuneo KC, Vredenburgh JJ, Sampson JH, Kirkpatrick JP (2012) Stereotactic radiosurgery and bevacizumab for recurrent glioblastoma multiforme. J Natl Compr Canc Netw 10:695-699

91. Gutin PH, Iwamoto FM, Beal K, Mohile NA, Karimi S, Hou BL, Lymberis S, Yamada Y, Chang J, Abrey LE (2009) Safety and efficacy of bevacizumab with hypofractionated stereotactic irradiation for recurrent malignant gliomas. Int J Radiat Oncol Biol Phys 75:156-63

92. Lai A, Tran A, Nghiemphu PL, et al (2011) Phase II study of bevacizumab plus temozolomide during and after radiation therapy for patients with newly diagnosed glioblastoma multiforme. J Clin Oncol 29:142-148

93. Vredenburgh JJ, Desjardins A, Kirkpatrick JP, et al (2012) Addition of bevacizumab to standard radiation therapy and daily temozolomide is associated with minimal toxicity in newly diagnosed glioblastoma multiforme. Int J Radiat Oncol Biol Phys 82: 58-66

94. Vredenburgh JJ, Desjardins A, Reardon DA, et al (2011) The addition of bevacizumab to standard radiation therapy and temozolomide followed by bevacizumab, temozolomide, and irinotecan for newly diagnosed glioblastoma. Clin Cancer Res 17:4119-24

95. Wen PY, Macdonald DR, Reardon DA, et al (2010) Updated response assessment criteria for high-grade gliomas: Response assessment in neuro-oncology working group. J Clin Oncol 28: 1963-1972

96. Chinot OL, Macdonald DR, Abrey LE, Zahlmann G, Kerloeguen Y, Cloughesy TF (2013) Response assessment criteria for glioblastoma: practical adaptation and implementation in clinical trials of antiangiogenic therapy. Curr Neurol Neurosci Rep 13:347

97. Hegi ME, Diserens A-C, Gorlia T, et al (2005) MGMT gene silencing and benefit from temozolomide in glioblastoma. $\mathrm{N}$ Engl $\mathrm{J}$ Med 352:997-1003

98. DeLay M, Jahangiri A, Carbonell WS, Hu Y-L, Tsao S, Tom MW, Paquette J, Tokuyasu TA, Aghi MK (2012) Microarray analysis verifies two distinct phenotypes of glioblastomas resistant to antiangiogenic therapy. Clin Cancer Res 18:2930-42 
99. Lu KV, Bergers G (2013) Mechanisms of evasive resistance to anti-VEGF therapy in glioblastoma. CNS Oncol 2:49-65

100. Casanovas O, Hicklin DJ, Bergers G, Hanahan D (2005) Drug resistance by evasion of antiangiogenic targeting of VEGF signaling in late-stage pancreatic islet tumors. Cancer Cell 8:299-309

101. Rigamonti N, Kadioglu E, Keklikoglou I, Rmili CW, Leow CC, de Palma M (2014) Role of angiopoietin-2 in adaptive tumor resistance to VEGF signaling blockade. Cell Rep 8:696-706

102. Huang D, Ding Y, Zhou M, Rini BI, Petillo D, Qian C, Kahnoski R, Futreal PA, Furge KA, Teh BT (2010) Interleukin-8 mediates resistance to antiangiogenic agent sunitinib in renal cell carcinoma. Cancer Res 70:1063-71

103. Kloepper J, Riedemann L, Amoozgar Z, et al (2016) Ang-2/VEGF bispecific antibody reprograms macrophages and resident microglia to anti-tumor phenotype and prolongs glioblastoma survival. Proc Natl Acad Sci USA 113:4476-4481

104. Peterson TE, Kirkpatrick ND, Huang Y, et al (2016) Dual inhibition of Ang-2 and VEGF receptors normalizes tumor vasculature and prolongs survival in glioblastoma by altering macrophages. Proc Natl Acad Sci USA 113(16):440-5

105. Park J-S, Kim I-K, Han S, et al (2016) Normalization of tumor vessels by Tie2 activation and Ang2 inhibition enhances drug delivery and produces a favorable tumor microenvironment. Cancer Cell 30:953-967

106. Sitohy B, Nagy JA, Jaminet SCS, Dvorak HF (2011) Tumorsurrogate blood vessel subtypes exhibit differential susceptibility to anti-VEGF therapy. Cancer Res 71:7021-7028

107. Benjamin LE, Golijanin D, Itin A, Pode D, Keshet E (1999) Selective ablation of immature blood vessels in established human tumors follows vascular endothelial growth factor withdrawal. J Clin Invest 103:159-165

108. Piao Y, Liang J, Holmes L, Henry V, Sulman E, De Groot JF (2013) Acquired resistance to anti-VEGF therapy in glioblastoma is associated with a mesenchymal transition. Clin Cancer Res 19: 4392-4403

109. Lu K V, Chang JP, Parachoniak CA, et al (2012) VEGF inhibits tumor cell invasion and mesenchymal transition through a MET/ VEGFR2 complex. Cancer Cell 22:21-35

110. Jahangiri A, De Lay M, Miller LM, et al (2013) Gene expression profile identifies tyrosine kinase c-Met as a targetable mediator of antiangiogenic therapy resistance. Clin Cancer Res 19:1773-83

111. Lucio-Eterovic AK, Piao Y, De Groot JF (2009) Mediators of glioblastoma resistance and invasion during antivascular endothelial growth factor therapy. Clin Cancer Res 15:4589-4599

112. Huang Y, Goel S, Duda DG, Fukumura D, Jain RK (2013) Vascular normalization as an emerging strategy to enhance cancer immunotherapy. Cancer Res 73:2943-2948

113. De Groot JF, Piao Y, Tran H, et al (2011) Myeloid biomarkers associated with glioblastoma response to anti-VEGF therapy with aflibercept. Clin Cancer Res 17:4872-4881

114. Shojaei F, Wu X, Qu X, Kowanetz M, Yu L, Tan M, Meng YG, Ferrara N (2009) G-CSF-initiated myeloid cell mobilization and angiogenesis mediate tumor refractoriness to anti-VEGF therapy in mouse models. Proc Natl Acad Sci USA 106:6742-6747

115. Chung AS, Wu X, Zhuang G, et al (2013) An interleukin-17mediated paracrine network promotes tumor resistance to antiangiogenic therapy. Nat Med 19:1114-1123

116. Tripathi C, Tewari BN, Kanchan RK, Baghel KS, Nautiyal N, Shrivastava R, Kaur H, Bhatt MLB, Bhadauria S (2014) Macrophages are recruited to hypoxic tumor areas and acquire a pro-angiogenic M2-polarized phenotype via hypoxic cancer cell derived cytokines Oncostatin M and Eotaxin. Oncotarget 5:5350 68

117. Scholz A, Harter PN, Cremer S, et al (2015) Endothelial cellderived angiopoietin- 2 is a therapeutic target in treatment-naive and bevacizumab-resistant glioblastoma. EMBO Mol Med 8(1): 39-57

118. Buchroithner J, Pichler J, Marosi C, et al (2014) Vascular endothelia growth factor targeted therapy may improve the effect of dendritic cell-based cancer immune therapy. Int J Clin Pharmacol Ther 52:76-7

119. Reardon DA, Schuster J, Tran DD, et al (2015) ReACT: Longterm survival from a randomized phase II study of rindopepimut (CDX-110) plus bevacizumab in relapsed glioblastoma. J Clin Oncol 33:abstr 2009

120. Shrimali RK, Yu Z, Theoret MR, Chinnasamy D, Restifo NP, Rosenberg SA (2010) Antiangiogenic agents can increase lymphocyte infiltration into tumor and enhance the effectiveness of adoptive immunotherapy of cancer. Cancer Res 70:6171-80

121. Sulman EP, Won M, Blumenthal DT, et al (2013) Molecular predictors of outcome and response to bevacizumab (BEV) based on analysis of RTOG 0825 , a phase III trial comparing chemoradiation (CRT) with and without BEV in patients with newly diagnosed glioblastoma (GBM). J Clin Oncol 31:LBA2010

122. Sathornsumetee S, Cao Y, Marcello JE, Herndon JE, McLendon RE, Desjardins A, Friedman HS, Dewhirst MW, Vredenburgh JJ, Rich JN (2008) Tumor angiogenic and hypoxic profiles predict radiographic response and survival in malignant astrocytoma patients treated with bevacizumab and irinotecan. J Clin Oncol 26: 271-8

123. Lu-Emerson C, Snuderl M, Kirkpatrick ND, et al (2013) Increase in tumor-associated macrophages after antiangiogenic therapy is associated with poor survival among patients with recurrent glioblastoma. Neuro Oncol 15:1079-1087

124. Chen C, Huang R, MacLean A, Muzikansky A, Mukundan S, Wen PY, Norden AD (2013) Recurrent high-grade glioma treated with bevacizumab: Prognostic value of MGMT methylation, EGFR status and pretreatment MRI in determining response and survival. J Neurooncol 115:267-276

125. Lv S, Teugels E, Sadones J, et al (2011) Correlation between IDH1 gene mutation status and survival of patients treated for recurrent glioma. Anticancer Res 31:4457-4463

126. Batchelor TT, Gerstner ER, Emblem KE, et al (2013) Improved tumor oxygenation and survival in glioblastoma patients who show increased blood perfusion after cediranib and chemoradiation. Proc Natl Acad Sci USA 110:19059-64

127. Duda DG, Willett CG, Ancukiewicz M, et al (2010) Plasma soluble VEGFR-1 is a potential dual biomarker of response and toxicity for bevacizumab with chemoradiation in locally advanced rectal cancer. Oncologist 15:577-583

128. Duda DG, Kozin S V., Kirkpatrick ND, Xu L, Fukumura D, Jain RK (2011) CXCL12 (SDF1 $\alpha$ )-CXCR4/CXCR7 pathway inhibition: An emerging sensitizer for anticancer therapies? Clin Cancer Res 17:2074-2080

129. Tabouret E, Boudouresque F, Barrie M, et al (2014) Association of matrix metalloproteinase 2 plasma level with response and survival in patients treated with bevacizumab for recurrent high-grade glioma. Neuro Oncol 16:392-399

130. De Groot JF, Lamborn KR, Chang SM, et al (2011) Phase II study of aflibercept in recurrent malignant glioma: A North American brain tumor consortium study. J Clin Oncol 29:2689-2695

131. Sorensen AG, Batchelor TT, Zhang W-T, et al (2009) A "vascular normalization index" as potential mechanistic biomarker to predict survival after a single dose of cediranib in recurrent glioblastoma patients. Cancer Res 69:5296-5300

132. Pope WB, Kim HJ, Huo J, et al (2009) Recurrent glioblastoma multiforme: ADC histogram analysis predicts response to bevacizumab treatment. Radiology 252:182-189

133. Kothari PD, White NS, Farid N, Chung R, Kuperman JM, Girard HM, Shankaranarayanan A, Kesari S, McDonald CR, Dale AM (2013) Longitudinal restriction spectrum imaging is resistant to 
pseudoresponse in patients with high-grade gliomas treated with bevacizumab. AJNR Am J Neuroradiol 34:1752-7

134. Piludu F, Marzi S, Pace A, Villani V, Fabi A, Carapella CM, Terrenato I, Antenucci A, Vidiri A (2015) Early biomarkers from dynamic contrast-enhanced magnetic resonance imaging to predict the response to antiangiogenic therapy in high-grade gliomas. Neuroradiology 57:1269-1280

135. O'Connor JPB, Jackson A, Parker GJM, Roberts C, Jayson GC (2012) Dynamic contrast-enhanced MRI in clinical trials of antivascular therapies. Nat Rev Clin Oncol 9:167-177

136. Emblem KE, Mouridsen K, Bjornerud A, et al (2013) Vessel architectural imaging identifies cancer patient responders to antiangiogenic therapy. Nat Med 19:1178-83

137. Chen W, Delaloye S, Silverman DHS, et al (2007) Predicting treatment response of malignant gliomas to bevacizumab and irinotecan by imaging proliferation with $[18 \mathrm{~F}]$ fluorothymidine positron emission tomography: A pilot study. J Clin Oncol 25: 4714-4721

138. Harris RJ, Cloughesy TF, Pope WB, Nghiemphu PL, Lai A, Zaw T, Czernin J, Phelps ME, Chen W, Ellingson BM (2012) 18FFDOPA and $18 \mathrm{~F}-\mathrm{FLT}$ positron emission tomography parametric response maps predict response in recurrent malignant gliomas treated with bevacizumab. Neuro Oncol 14:1079-1089

139. Sorensen AG, Emblem KE, Polaskova P, et al (2012) Increased survival of glioblastoma patients who respond to antiangiogenic therapy with elevated blood perfusion. Cancer Res 72:402-407

140. Lorgis V, Maura G, Coppa G, Hassani K, Taillandier L, Chauffert B, Apetoh L, Ladoire S, Ghiringhelli F (2012) Relation between bevacizumab dose intensity and high-grade glioma survival: A retrospective study in two large cohorts. J Neurooncol 107:351358

141. Levin VA, Mendelssohn ND, Chan J, Stovall MC, Peak SJ, Yee JL, Hui RL, Chen DM (2015) Impact of bevacizumab administered dose on overall survival of patients with progressive glioblastoma. J Neurooncol 122:145-150

142. Weathers S-P, Han X, Liu DD, et al (2016) A randomized phase II trial of standard dose bevacizumab versus low dose bevacizumab plus lomustine (CCNU) in adults with recurrent glioblastoma. J Neurooncol 129:487-494

143. Reardon DA, Herndon JE, Peters KB, et al (2012) Bevacizumab continuation beyond initial bevacizumab progression among recurrent glioblastoma patients. Br J Cancer 107:1481-7

144. Tang TC, Man S, Xu P, Francia G, Hashimoto K, Emmenegger U, Kerbel RS (2010) Development of a resistance-like phenotype to sorafenib by human hepatocellular carcinoma cells is reversible and can be delayed by metronomic UFT chemotherapy. Neoplasia 12:928-940

145. Bennouna J, Sastre J, Arnold D, et al (2013) Continuation of bevacizumab after first progression in metastatic colorectal cancer (ML18147): A randomised phase 3 trial. Lancet Oncol 14:29-37

146. von Minckwitz G, Puglisi F, Cortes J, et al (2014) Bevacizumab plus chemotherapy versus chemotherapy alone as second-line treatment for patients with HER2-negative locally recurrent or metastatic breast cancer after first-line treatment with bevacizumab plus chemotherapy (TANIA): an open-label, randomised. Lancet Oncol 15:1269-78 